\title{
Specific Densified Regions in the Postlaminar Human Glaucomatous Optic Nerve
}

\author{
Christian Albrecht May*
}

Department of Anatomy, Faculty of Medicine Carl Gustav Carus, Technische Universität Dresden, Dresden, Germany

\begin{abstract}
Purpose: To describe 'densified regions' in human postlaminar optic nerves of donors with the clinical diagnosis of primary open angle glaucoma (POAG) and pseudoexfoliation glaucoma (PEXG).

Methods: Optic nerves of 25 eyes with POAG and of 19 eyes with PEXG were investigated. Clinical data and axon counts of Epon-embedded semi-thin sections were used to determine the stage of the disease. Ultrathin sections were performed of selected areas and studied with an electron microscope. Immunofluorescence staining of paraffin sections was performed including antibodies against glial fibrillary acidic protein (GFAP), neural cellular adhesion molecule, vimentin and laminin.

Results: Early changes in both types of glaucoma showed increased numbers of areas with a densified appearance. The lack of GFAP staining and of ultrastructurally light nuclei within these areas indicated loss of astroglia.

Conclusion: For the first time, a special type of neurodegeneration in optic nerves with mild glaucomatous changes is described.
\end{abstract}

Keywords: Astrocytes, degeneration, glaucoma, glial fibrillary acidic protein (GFAP), optic nerve.

\section{INTRODUCTION}

It is a known but not widely mentioned fact that within the nerve fiber bundles of the retrolaminar optic nerve densified regions can occur, independent of the topographic location of the optic nerve within the orbit. These regions were first noted by E. Fuchs [1] and subsequently shown by Kolmer [2]. Originally discussed by Fuchs as some type of atrophy, the more recent interpretation by Kolmer was some artifact of preservation. Since this phenomenon has only been described in human optic nerve sections and seems not to appear in the usually used animal models applied for optic nerve studies, it became forgotten in the literature.

Examining numerous human retrolaminar optic nerve semithin cross sections over the last 15 years by light microscopy, occasionally densified regions could be observed in otherwise healthy eyes (unpublished results). Electron microscopic sections of these densified regions showed the normal appearance of optic nerve tissue, preserved axons and myelin sheaths, surrounded by astrocyte processes. The only difference to the surrounding neuronal tissue was a closer arrangement of myelinated nerve fibres. Immunohistochemical staining of these regions revealed a normal staining for GFAP within these densifications. Therefore, in the normal human optic nerve these regions represent morphologically intact densified myelinated axons with an unknown etiology of the densification process.

*Address correspondence to this author at the Department of Anatomy, Medizinische Fakultät Carl Gustav Carus, Fetscherstr. 74, 01307 Dresden, Germany; Tel: +49-351-458-6105; Fax: +49-351-458-6303;

E-mail: Albrecht.May@tu-dresden.de
In the present report densified regions were studied in optic nerves of glaucomatous eyes to test if these regions represent some differences to the normal optic nerve.

\section{MATERIAL AND METHODS}

\section{Tissue Samples}

Twenty-five optic nerves (ON) from 14 human donors with the clinical diagnosis of POAG or glaucoma suspect and nineteen ONs from 11 human donors with the clinical diagnosis of PEX syndrome, observed by slit lamp biomicroscopy, were studied. Clinical data of these eyes is provided in Table $\mathbf{1}$ and was partly published previously [3]; research followed the tenets of the Declaration of Helsinki.

After enucleation of the eyes 2-12 hours post mortem, the ONs were immersion fixed in a mixture of $1-2.5 \%$ glutaraldehyde and $2.5-4 \%$ formaldehyde for several days.

\section{Processing and Staining}

Small ON cross sections were rinsed in cacodylate buffer ( $\mathrm{pH}$ 7.4), postfixed in $1 \%$ osmium tetroxide, dehydrated and embedded in Epon. Semi-thin cross sections were stained with toluidine blue. Ultrathin sections of selected areas were stained with uranyl acetate and lead citrate, and viewed with a Zeiss EM 902 (Zeiss, Oberkochen, Germany) electron microscope.

For immunohistochemistry, optic nerve cross sections were rinsed in phosphate buffered saline (PBS, pH 7.2-7.4), dehydrated, and embedded in paraffin. Sections ( $5 \mu \mathrm{m}$ thick) were placed on poly-L-lysine coated glass slides, 
Table 1. Clinical data of the eyes investigated.

Clinical data of the Primary open angle glaucoma eyes

\begin{tabular}{|c|c|c|c|c|c|c|}
\hline Age (Years)/ Sex & Duration (Years) & Eye & IOPmax (mmHg) & IOPther (mmHg) & C/D ratio & Visual Field \\
\hline \multirow{2}{*}{$76 / \mathrm{m}$} & \multirow{2}{*}{14} & OD & & 16 & 0.2 & normal \\
\hline & & OS & & 16 & 0.2 & normal \\
\hline \multirow{2}{*}{$65 / \mathrm{m}$} & \multirow{2}{*}{8} & OD & 35 & 22 & 0.4 & normal \\
\hline & & OS & 35 & 22 & 0.9 & Sup arc \\
\hline \multirow{2}{*}{$63 / \mathrm{f}$} & \multirow{2}{*}{13} & OD & 34 & 20 & 0.1 & normal \\
\hline & & OS & 34 & 22 & 0.1 & normal \\
\hline $80 / \mathrm{m}$ & 23 & OS & 25 & 20 & 0.4 & normal \\
\hline \multirow{2}{*}{$89 / \mathrm{f}$} & \multirow{2}{*}{10} & OD & 28 & 15 & $?$ & $?$ \\
\hline & & OS & 29 & 15 & $?$ & $?$ \\
\hline \multirow{2}{*}{$69 / \mathrm{m}$} & \multirow{2}{*}{2} & OD & 26 & $?$ & 0.2 & normal \\
\hline & & OS & 26 & $?$ & 0.2 & normal \\
\hline \multirow{2}{*}{$81 / \mathrm{m}$} & \multirow{2}{*}{5} & OD & 26 & 14 & 0.8 & normal \\
\hline & & OS & 26 & 16 & 0.2 & Sup arc \\
\hline \multirow{2}{*}{$86 / \mathrm{f}$} & \multirow{2}{*}{7} & OD & 32 & 17 & 0.7 & Sup arc \\
\hline & & OS & 32 & 17 & 0.6 & normal \\
\hline \multirow{2}{*}{$84 / \mathrm{f}$} & \multirow{2}{*}{13} & OD & 25 & 12 & 0.7 & $?$ \\
\hline & & OS & 22 & 13 & 0.6 & $?$ \\
\hline $74 / \mathrm{f}$ & 5 & OD & 30 & 22 & 0.6 & normal \\
\hline \multirow{2}{*}{$60 / \mathrm{m}$} & \multirow{2}{*}{5} & OD & $?$ & 22 & 0.3 & Sup arc \\
\hline & & OS & $?$ & 16 & 0.3 & constr \\
\hline $63 / \mathrm{m}$ & $?$ & OS & 26 & 19 & $?$ & $?$ \\
\hline \multirow{2}{*}{$80 / \mathrm{f}$} & \multirow{2}{*}{5} & OD & 28 & 18 & 0.6 & normal \\
\hline & & OS & 26 & 14 & 0.6 & normal \\
\hline \multirow{2}{*}{$72 / f$} & \multirow{2}{*}{14} & OD & $?$ & $?$ & $?$ & $?$ \\
\hline & & OS & $?$ & $?$ & $?$ & $?$ \\
\hline
\end{tabular}

Clinical data of the Pseudoexfoliation glaucoma eyes

\begin{tabular}{|c|c|c|c|c|c|c|}
\hline Age (Years)/ Sex & Duration (Years) & Eye & IOPmax (mmHg) & IOPther (mmHg) & C/D Ratio & Visual Field \\
\hline $93 / \mathrm{f}$ & 7 & OS & 30 & 23 & 0.7 & normal \\
\hline $83 / \mathrm{f}$ & 1 & OD & 16 & 16 & 0.4 & normal \\
\hline \multirow{2}{*}{$87 / f$} & \multirow{2}{*}{2} & OD & 20 & 14 & 0.5 & normal \\
\hline & & OS & 19 & 16 & 0.6 & normal \\
\hline \multirow{2}{*}{$79 / f$} & \multirow{2}{*}{4} & OD & 28 & 19 & 0.3 & normal \\
\hline & & OS & 31 & 21 & 0.3 & normal \\
\hline $93 / \mathrm{f}$ & 7 & OS & 45 & 25 & 0.2 & normal \\
\hline \multirow{2}{*}{$100 / \mathrm{m}$} & \multirow{2}{*}{12} & OD & 29 & 15 & 0.9 & Sup arc \\
\hline & & OS & 33 & 15 & 0.9 & Sup arc \\
\hline \multirow{2}{*}{$86 / \mathrm{m}$} & \multirow{2}{*}{10} & OD & 21 & 17 & 0.3 & normal \\
\hline & & OS & 18 & 15 & 0.9 & normal \\
\hline \multirow{2}{*}{$89 / \mathrm{m}$} & \multirow{2}{*}{5} & OD & 46 & 20 & 0.8 & blind \\
\hline & & OS & 26 & 16 & $?$ & central \\
\hline \multirow{2}{*}{$98 / \mathrm{m}$} & \multirow{2}{*}{7} & OD & 22 & 13 & 0.2 & constr \\
\hline & & OS & 35 & 17 & 0.2 & Sup arc \\
\hline \multirow{2}{*}{$71 / \mathrm{f}$} & \multirow{2}{*}{20} & OD & 22 & 17 & 0.3 & normal \\
\hline & & OS & 45 & 30 & 1.0 & Sup arc \\
\hline \multirow{2}{*}{$88 / \mathrm{m}$} & \multirow{2}{*}{9} & OD & 25 & 16 & 0.3 & $?$ \\
\hline & & OS & 27 & 18 & 0.6 & $?$ \\
\hline
\end{tabular}

$\mathrm{m}=$ male, $\mathrm{f}=$ female; $\mathrm{OD}=$ right eye, $\mathrm{OS}=$ left eye; IOPmax $=$ highest intraocular pressure measured; IOPther $=$ mean intraocular pressure during therapy; $\mathrm{C} / \mathrm{D}$ ratio $=$ cup/disc ratio; Sup arc $=$ superior arcuate scotoma, constr $=$ constricted visual field . 
deparaffinized and rehydrated. The sections were incubated with the primary antibody over night at $4^{\circ} \mathrm{C}$ in a moist chamber. The primary antibodies used were directed against glial fibrillar acidic protein (GFAP; 1:200, Biogenex, San Ramon, CA, USA), neural cellular adhesion molecule (NCAM; 1:100, Sigma, Deisenhofen, Germany), vimentin (1:200; Dako, Glostrup, Denmark), and laminin (1:200; Sigma). After rinsing in PBS, the sections were incubated with an appropriate fluorescent labeled secondary antibody for one hour at room temperature, rinsed again, and mounted with glycerin jelly.

Unfortunately, due to the fixation of the tissue the number of antibodies was restricted, and numerous additional antibodies which were tested for further characterization did not show sufficient staining.

\section{Evaluation}

Axon count was calculated as described previously [3]. In brief, the axons in randomly selected areas of the optic nerve were counted by light microscopy and the total number calculated by projecting the counts to the total cross section area. Due to the number of myelinated nerve fibers in single cross sections, the optic nerves were grouped into three stages of severity. Mild optic neuropathy was considered in optic nerves with axon counts more than 500,000 . In the group of moderate optic neuropathy, sections with axon counts between 100,000 and 500,000 were included. Severe stages of optic neuropathy contained less than 100,000 myelinated axons.

The densified areas in the whole optic nerve were counted and measured by light microscopy. Immunohistochemical staining was investigated using a Leica Aristoplan fluorescence microscope.

\section{RESULTS}

\section{Densified Regions: Frequency of Appearance}

Most of the densified regions were observed in optic nerve cross sections of donors with mild optic neuropathy (Table 2): they were located within the neuronal tissue of the optic nerve and did not reach the rim of the optic nerve (pia mater). At places, they filled in almost a complete bundle reaching towards the connective tissue septae within the nerve (Fig. 1). Their diameter ranged between 120 and 300 $\mu \mathrm{m}$; their number varied greatly within the single nerves, even between the right and left nerve of a single donor. The densified regions could be identified in consecutive sections; the tissue samples, however, did not allow a longitudinal reconstruction within the optic nerve. The densified regions were found regardless of the section distance towards the lamina cribrosa, the distance varying between $2-20 \mathrm{~mm}$.

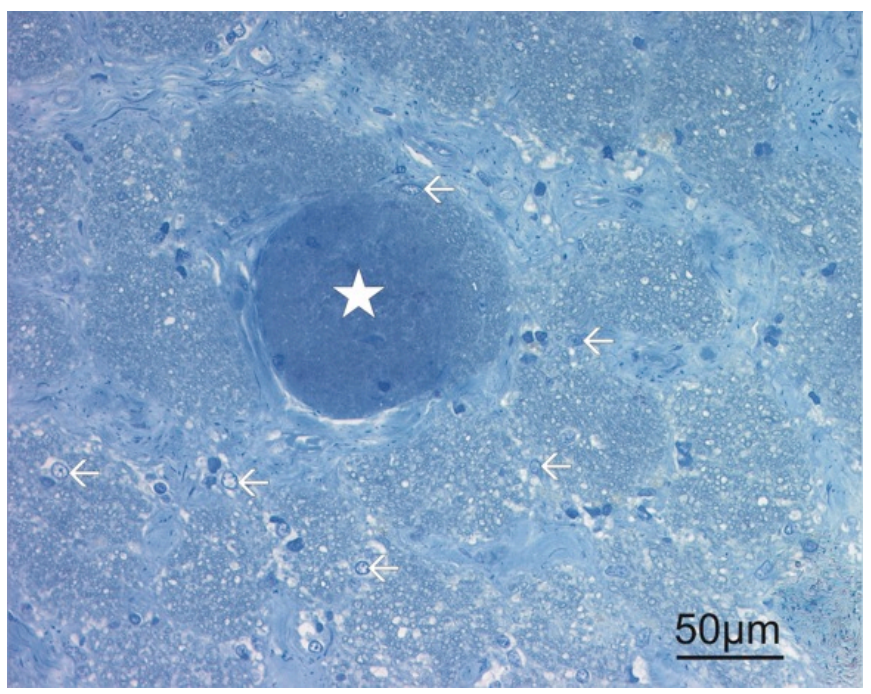

Fig. (1). Toluidine blue stained semi thin section of an optic nerve with mild optic neuropathy ( 80 years old female donor with POAG). Note the densified region marked with a star. Within this region, some dense nuclei can be observed. There was a complete lack of light nuclei (arrows outside of the densified region) as an indication for astrocytes.

In optic nerve cross sections defined as moderate optic neuropathy, still some densified regions could be observed in POAG, but their number was less frequent (Table 2). Their size and localization was the same as in mild optic neuropathy. The regions were never seen in contact to gliotic areas with complete axonal degeneration.

Table 2. Number and occurrence of densified regions in different stages of primary open angle and pseudoexfoliation glaucoma optic neuropathy.

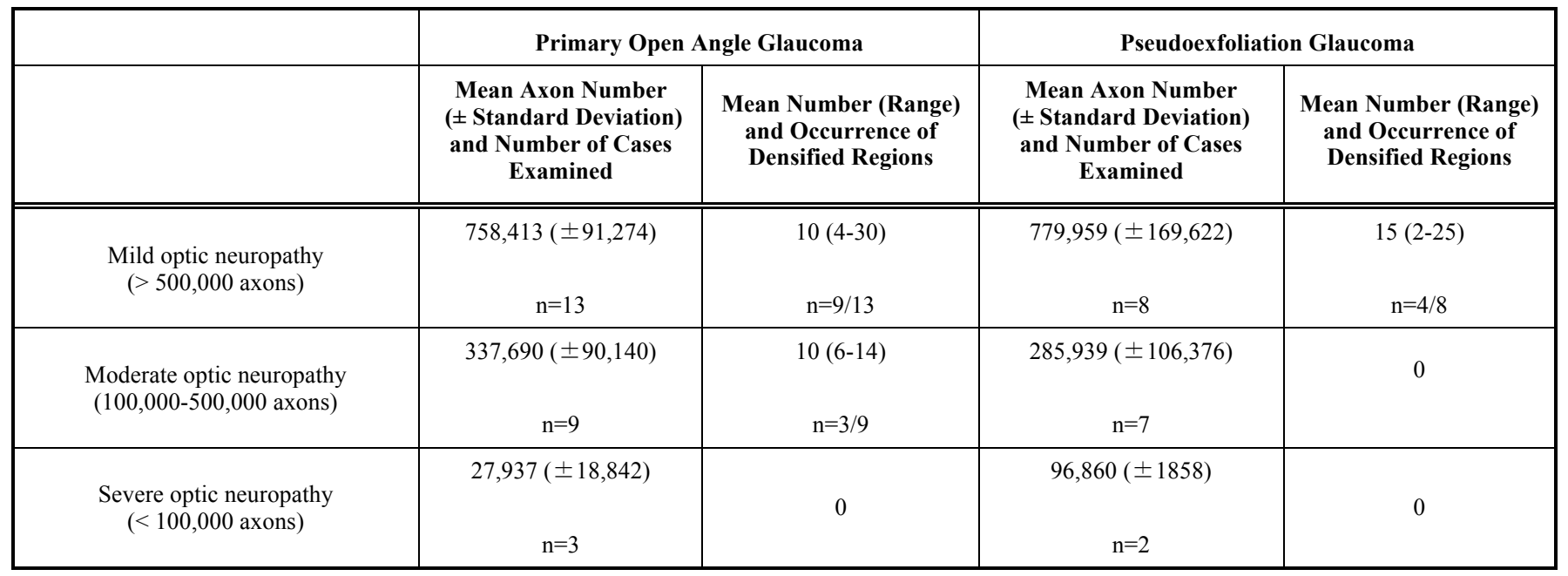


No densified regions were present in severe stages of optic neuropathy; here an increase in gliotic reaction and a profound degeneration of axons dominated most areas of the optic nerve cross sections.

\section{Densified Regions: Immunohistochemical Characterization}

Immunohistochemical staining for GFAP, the most general marker for astrocytes, revealed, that within these regions, no GFAP positive cells were present (Fig. 2a). The surrounding neural tissue showed the regular appearance of GFAP staining as seen in non-glaucomatous optic nerves. The staining intensity next to the GFAP-free regions did not show increased values and no encapsulating processes of the astrocytes. Surrounding astrocytes, therefore, seemed not activated by the process of densification.
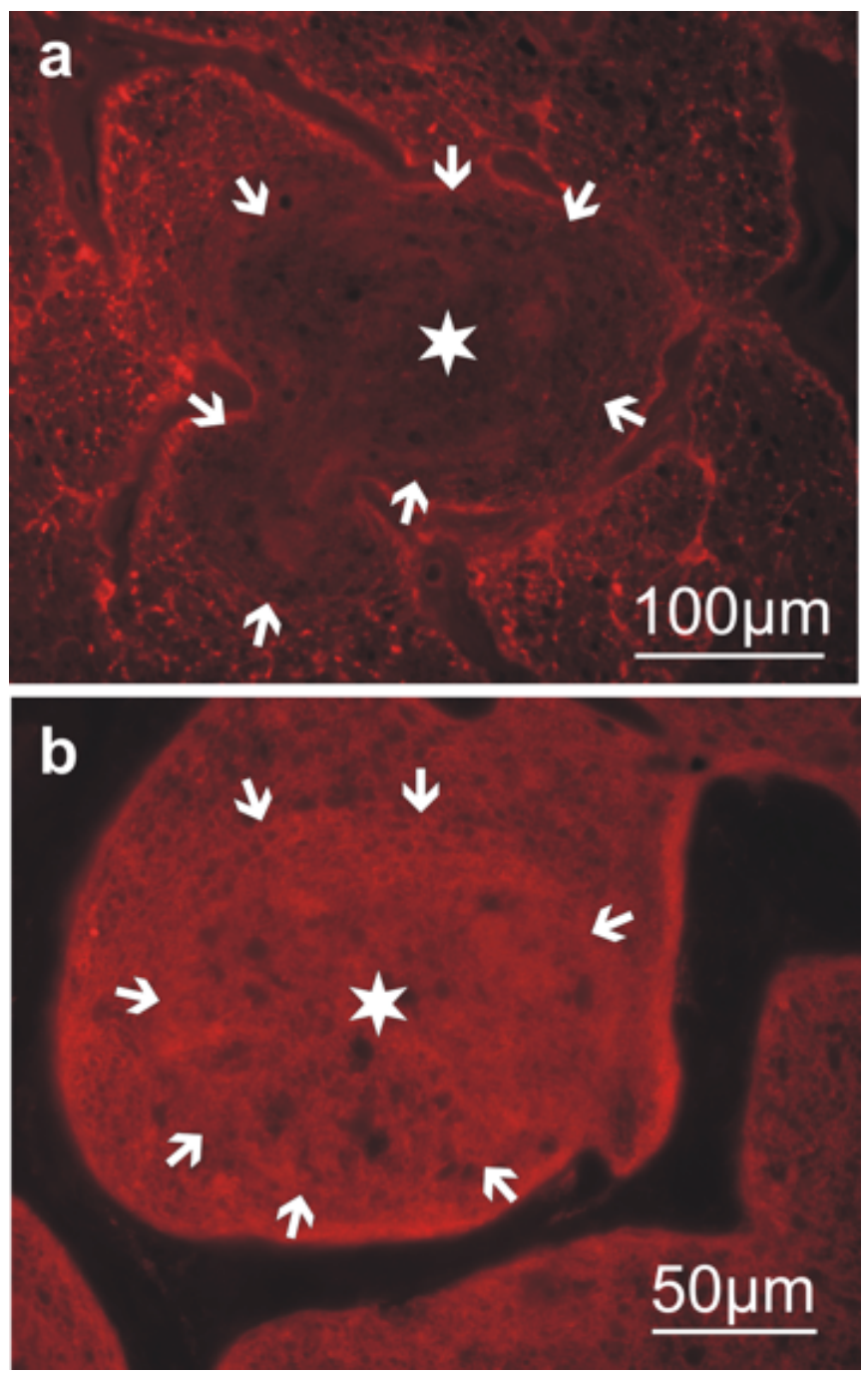

Fig. (2). Immunohistochemistry of optic nerve cross sections with mild optic neuropathy (60 years old male donor with POAG). The densified region is marked with a star, the rim of the region with numerour arrows. a) Staining with antibodies against glial fibrillary acidic protein (GFAP) reveals a lack of staining within the densified area and no specific accent at the rim of the region or within the surrounding neuronal tissue. b) Staining with antibodies against neural cellular adhesion molecule (N-CAM) shows a diffuse staining within the densified area similar to the surrounding neuronal tissue.
Staining for NCAM, a marker for various cell types derived from the neuroectoderm (mainly glial cells but also some neurons), revealed positive cells allover the neuronal tissue of optic nerve cross sections including the densified regions (Fig. 2b). A similar staining was observed using antibodies against vimentin (data not shown).

Laminin staining was seen at the rim of the connective tissue within the optic nerve but not within the neuronal tissue or in contact with the densified regions (data not shown).

\section{Densified Regions: Ultrastructural Morphology}

Electron microscopy showed numerous small myelin rings (presumably small axons) within the densified regions. They were surrounded by an electron-dense cytoplasm containing degenerated myelin forms (Fig. 3). Normal-sized axons were absent in the densified regions. Nuclei present within the densified areas showed dense chromatin, usually seen in either oligodendrocytes or microglia. The light nuclei of astrocytes were never observed within these regions. The rim of the densified regions showed no specific cellular or extracellular structure.

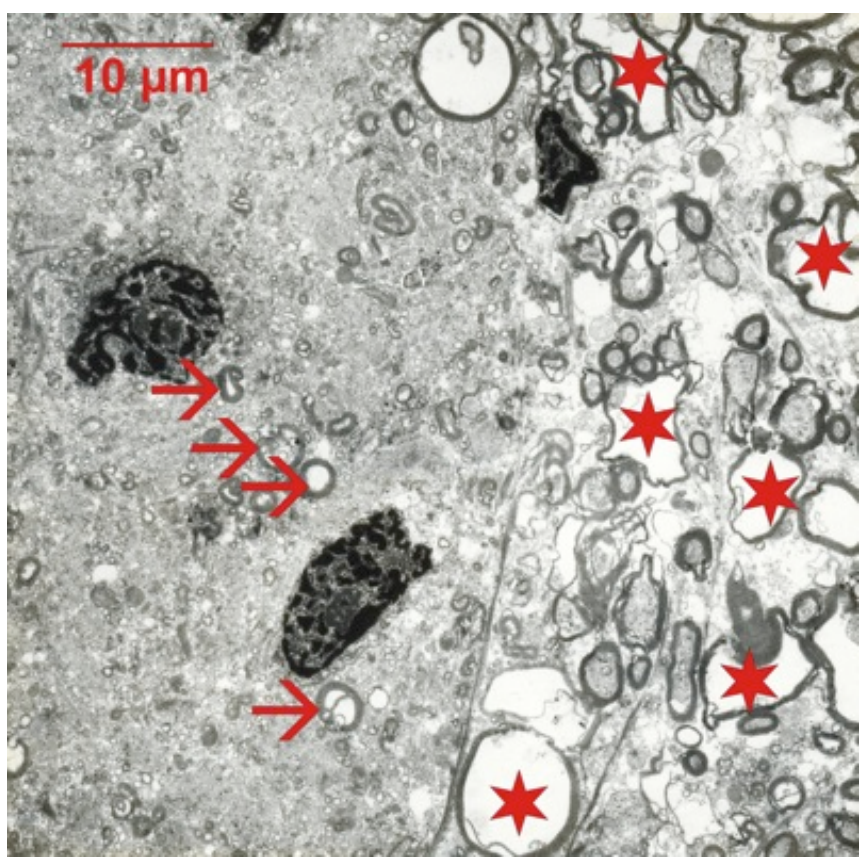

Fig. (3). Electron microscopic micrograph of the optic nerve (63 years old male donor with POAG) at the rim of a densified area. Note the different appearance between densified area (left) and the normal neuronal tissue (right). In the densified areas, some small axons and their myelin sheath seem to be preserved (arrows), but their density is low and they are surrounded by an electron dense cytoplasm with numerous myelin debris. Larger axons (stars) are only present outside of the densified areas. The dark dense nuclei indicate either oligodendrocytes or microglia.

\section{DISCUSSION}

While densified regions in the optic nerve with unknown origin can occur sporadic in otherwise healthy eyes, densified regions occur frequently in early stages of glaucomatous optic neuropathy. They are located within healthy neuronal tissue and are not separated by specific morphological features. In contrast to densified regions in 
non-pathologic optic nerves, the densified areas in glaucomatous optic neuropathy showed a lack of GFAP staining without affecting the surrounding neuronal tissue.

While experimental hypoxia lead only to an early, reversible reduction of GFAP [4], decreased GFAP was also seen in chronic swelling and vacuolation of white matter astroglia (clasmatodendrosis) in Alzheimer's disease [5]. A true lack of GFAP staining has been described in severe focal experimental cerebral ischemia, however with increase of GFAP in the surrounding tissue [6]. An early astrocyte reaction in the optic nerve has also been described for inflammatory diseases [7]. The findings in the densified regions of glaucomatous optic nerves, however, contradict the expected GFAP (astrocyte) activation, which is frequently seen in more severe stages of glaucomatous optic neuropathy [8]. Therefore, a specific process seems to take place in early stages of glaucomatous neuropathy: the astrocytes seem to retrain their processes from regions of local activity (possibly pre-degeneration) without forming a wall around these regions. Interestingly, there was no specific correlation of early optic neuropathy with any of the few clinical data available. Since the densified regions were only small compared to the optic nerve diameter, these activities per se seem not to influence the clinical process.

Within the densified regions of glaucomatous optic nerves, vesicular myelin formations and irregularities were regularly present showing similarities to nerve degeneration in the peripheral nervous system: early stages of Walleriantype degeneration show vesicular myelin formations, replacing the axon by tubulovesicular structures [9]. Although this process is described for single nerve fibers, it might take place in focal groups of neighboring neurons in the optic nerve, too.

Since previous work could show vascular changes (loss and occlusion of capillaries) in the postlaminar optic nerve of glaucomatous eyes [3], these changes might support the importance of ischemia in the progress of glaucomatous optic nerve pathology. Acute anoxia, however, does not lead primarily to changes in the myelin sheath [10]. Therefore, the densified regions in early stages of glaucomatous optic neuropathy need more attention and might broaden the understanding of white matter degeneration.

\section{CONFLICT OF INTEREST}

The author confirms that this article content has no conflict of interest.

\section{ACKNOWLEDGEMENTS}

The author thanks Elke Lütjen-Drecoll and the late Doug Johnson for providing the material.

\section{REFERENCES}

[1] Fuchs E. Die periphere Atrophie des Sehnerven. Graefes Arch Clin Exp Ophthalmol 1885; 31: 177-200.

[2] Kolmer W, Lauber H. Handbuch der mikroskopischen Anatomie. Berlin: Springer, 1936; 3: p.2.

[3] Gottanka J, Kuhlmann A, Scholz M, Johnson DH, Lütjen-Drecoll E. Pathophysiologic changes in the optic nerves of eyes with primary open angle and pseudoexfoliation glaucoma. Invest Ophthalmol Vis Sci 2005; 46: 4170-81.

[4] Martin LJ, Brambrink AM, Lehmann C, et al. Hypoxia-ischemia causes abnormalities in glutamate transporters and death of astroglia and neurons in newborn striatum. Ann Neurol 1997; 42: 335-48.

[5] Tomimoto H, Akiguchi I, Wakita H, Suenaga T, Nakamura S, Kimura J. Regressive changes of astroglia in white matter lesions in cerebrovascular disease and Alzheimer's disease patients. Acta Neuropathol 1997; 94: 146-52.

[6] Cheung WM, Wang CK, Kuo JS, Lin TN. Changes in the level of glial fibrillary acidic protein (GFAP) after mild and severe focal cerebral ischemia. Chin J Physiol 1999; 42: 227-35.

[7] Sharma R, Fischer MT, Bauer J, et al. Inflammation induced by innate immunity in the central nervous system leads to primary astrocyte dysfunction followed by demyelination. Acta Neuropathol 2010; 120: 223-36.

[8] Varela HJ, Hernandez MR. Astrocyte responses in human optic nerve head with primary open-angle glaucoma. J Glaucoma 1997; 6: 303-13.

[9] Liberski PP, Yanagihara R, Gibbs CJ Jr, Gajdusek DC. White matter ultrastructural pathology of experimental Creutzfeldt-Jakob disease in mice. Acta Neuropathol (Berl) 1989; 79: 1-9.

[10] Waxman SG, Black JA, Stys PK, Ransom BR. Ultrastructural concomitants of anoxic injury and early post-anoxic recovery in rat optic nerve. Brain Res 1992; 574: 105-19. 